\title{
Light avoidance in normal rats and rats with primary visual system lesions*
}

\author{
IAN Q. WHISHAW \\ University of Lethbridge, Lethbridge, Alberta, Canada
}

\begin{abstract}
The light-avoidance behavior of normal rats and rats with primary visual system lesions was examined in a differentially illuminated two-choice chamber over 28-58 days. Light avoidance in normal rats was distributed as a $\mathrm{J}$ curve, with some rats spending little time in the light and some rats spending up to $12 \mathrm{~h}$ each day in the light. Normal rats ate in the dark compartment, hoarded food to the dark, but defecated in the lighted compartment. Enucleation abolished light avoidance and the other behaviors. Neither small nor large posterior neocortex lesions affected light avoidance or other behaviors. Avoidance behavior was also unchanged following superior colliculus lesions and pretectal lesions, but was increased by lateral geniculate lesions, posterior thalamus lesions or combined posterior neocortex and hippocampal lesions, and posterior neocortex and superior colliculus lesions. The results do not support previous suggestions that primary optic-center lesions decrease light avoidance in the rat.
\end{abstract}

It is well known that if a rat is given a free choice between a lighter or darker area, it will move to and spend a major portion of its time in the darker area (Crozier \& Pincus, 1926; Keller, 1941; Keller \& Oberlin, 1942; Turner, 1935; Hanson, 1965): This finding has been interpreted as indicating that the rat has an aversion to light (Abelman \& Morgan, 1943; Altman, 1962). A number of investigations have been undertaken which seem to show that removal of part or all of visual cortex or subcortical visual structures leads to a reduction in light-aversion tendencies (Altman, 1962; Bauer, 1968; Horel, Bettinger, Royce, \& Meyer, 1966; Parker, Erickson, \& Treichler, 1968; Parker \& Treichler, 1973; Spear \& Braun, 1969) or alternately to an increase in light aversion (Abelman \& Morgan, 1943). Many of the effects in the discrimination tests may be situation specific (Parker \& Treichler, 1973; Stratton, 1971; Stratton \& Edwards, 1971). However, the free-choice tests (Altman, 1962; Parker \& Treichler, 1973) may merit further examination, since the test durations were relatively short (10-15 min) and periods as long as $3 \mathrm{~h}$ may be required before valid light-aversion results are obtained (Keller \& Oberlin, 1942). In the present study, rats were placed in a differentially illuminated two-compartment box for 14 days, received a lesion, and then were tested for a further 14-30 days. Measures were of light avoidance, activity levels, eating behavior, food hoarding, and defecation behavior.

\section{METHODS}

\section{Subjects}

The experiments were performed with 114 adult male hooded rats obtained from Canadian Animal Breeding Farms, La Prairie, Quebec. During the experiments, the animals were housed individually and had free access to water and food (standard blended calf pellets). When special feeding was required postoperatively, 20-60 cc of a liquid diet previously described by Teitelbaum and Epstein (1962) was given daily by intragastric route.

*This research was supported by Canadian National Research Grant A-8273 and University of Lethbridge Grant 28-1108.

\section{Surgical Procedure}

Neocortical lesions and combined neocortical-subcortical lesions were produced using the suction technique while the animals were deeply anesthetized with pentobarbital sodium (Nembutal). The skull was removed over the area to be aspirated and the underlying tissue carefully removed. Among animals which received visual neocortex removal, the size of the lesion was varied so that in some animals the entire posterior neocortex was removed posterior to bregma and laterally to the rhinal fissure, while in other animals the lesions were restricted to the striate cortex (Area 17) area as indicated by Zeman and Innes (1963). Similar aspiration lesions were made to produce decorticate animals, animals with combined lesions removing neocortex and hippocampus, neocortex and superior colliculus, and neocortex, hippocampus, and superior colliculus.

Subcortical electrolytic lesions were made by passing a cathodal current through both tips of a bipolar Nichrome electrode, which was insulated except for the cross section of the tips. The current intensity was $2.5 \mathrm{~mA}$, but duration varied between 20 and $60 \mathrm{sec}$, depending on the size of the lesion required. The circuit was completed by a rectal probe.

\section{Apparatus}

Twelve, two-compartment "tilt boxes" were used. The boxes consisted of two compartments, $20.3 \times 24.1 \mathrm{~cm}$ wide and $25.4 \mathrm{~cm}$ deep, with $7.6 \times 10.1 \mathrm{~cm}$ openings between the two compartments. The floor consisted of $3.2-\mathrm{mm}$ brass rods spaced $1.8 \mathrm{~cm}$ apart. The floor was suspended just under the partition on a fulcrum so that it could tilt slightly, activating a microswitch, which in turn activated a clock, counter, and Esterline-Angus 20-channel chart event recorder. One-half of the box was covered by a clear Plexiglas lid, permitting constant illumination of $100 \mathrm{fc}$, while the other side of the box was covered, permitting no overhead light entry, and had an ambient illumination of $3 \mathrm{fc}$ from light reflected beneath the box through the grids. A water bottle was located at each end of the box, and six pellets of food were placed in each half of the box each day. A fresh paper towel was placed beneath the box daily to catch food and defecation droppings.

\section{Procedure}

Procedure varied slightly in the different experiments (an experiment consisted of testing a group of 4-16 rats prior to and following a particular lesion), but the general procedure consisted of placing each rat into a box for 14 days, performing surgery, and then returning the animal to the apparatus for a further 14-30 days. When placed in the box, they were always placed in the lighted compartment, where they could stay or enter the darker compartment. In all experiments, before the rat was finally removed from the box, the lid was reversed for 2-10 


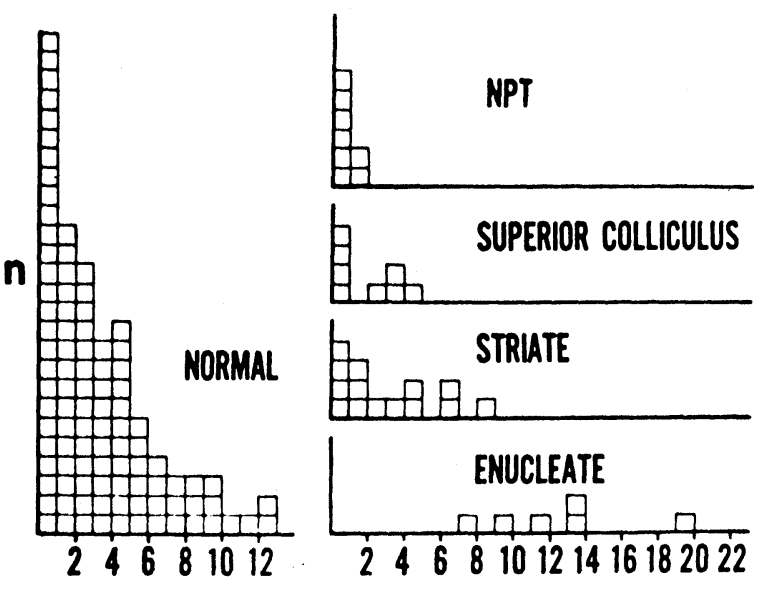

HOURS IN LIGHT

Fig. 1. Light avoidance shown by normal rats, enucleated rats, rats with large posterior neocortex lesions (striate), superior colliculus lesions, or posterior thalamic (NPT) lesions. Each square represents one animal. Note the $\mathbf{J}$ distribution in normal rats and also the similar distribution in striate, and superior colliculus groups. Note that avoidance is increased by NPT lesions but decreased by enucleation.

days, reversing the light and dark sides, to determine if the rat's preference would reverse. In cases where animals did not eat after surgery, data were collected each day, but the formal 14-day period did not begin until an animal had resumed feeding. Throughout the tests, a record was made of: (a) Light avoidance-A permanent record was kept of the daily time a rat spent in the light, both on the chart recorder, which indicated the times of day a rat entered the light compartment, and on a clock which indicated total time in the light compartment. (b) Food consumption-Each day, six pellets of food were placed in each light and dark compartment, and a record was made of where rats ate by estimates from the location of crumbs on the paper benea th the grids. (c) Food hoarding-A record was made of the location of the food pellets within the box each day to see if the animals carried the pellets from one box to the other. (d) Defecation-During the experiments, it became apparent that animals preferred to defecate in either the light or dark compartment. Therefore, a record was made by daily counts of the number of droppings beneath each compartment each day.

\section{Histological Procedure}

At the conclusion of the experiments, the placement of lesions was confirmed using standard histological techniques. The animals were sacrificed with an overdose of pentobarbitone sodium, and a saline and a $20 \%$ Formalin solution was perfused through the heart. The brains were removed and stored in $10 \%$ Formalin. The brains were then frozen, sectioned at 40 microns, mounted, and stained with thionin for examination.

\section{RESULTS}

\section{Control Measures}

Two control measures were used to determine if time spent in a compartment of a box was reflecting an animal's response to light or to other factors, such as compartment preference, position, etc. (1) Nine rats were placed in a box for 14 days, then the lid was reversed, reversing the lighted compartment for 14 days, and finally the animals were moved to a second box for 14 more days. There was no significant change in individual animal's behavior across the three conditions. Three animals which spent between 100 and $600 \mathrm{~min}$ in the light each day maintained this behavior in each condition, while six animals which spent less than $100 \mathrm{~min}$ daily in the light maintained this behavior. (2) In the second test, six rats received the 14-day test, were blinded by enucleation, and tested for 14 more days. In the normal condition, the animals spent a mean of $179 \pm 91 \mathrm{~min}$ in the light daily. Following enucleation, the mean daily time increased to near chance $682 \pm 203 \mathrm{~min}$, a highly significant increase $(\mathrm{t}=$ $7.10, p<.001)$. Observation of individual rats' behavior indicated that after enucleation they moved from side to side in the boxes in what appeared to be a haphazard way, while preoperatively they spent the major portion of each day in the dark compartment, making only brief periodic excursions into the light.

\section{Light Avoidance}

A summary of the mean daily time spent in the light for each of 100 normal animals during the 14-day test is shown in Fig. 1. The distribution of behavior approximates a $\mathbf{J}$ curve. Most animals spent very little time in the light (mean $=128 \pm 178 \mathrm{~min}$ ), but a few animals spent as long as $12 \mathrm{~h}$ in the light each day. The behavior of individual rats was also remarkably stable, varying by as little as a few minutes a day over the 14-day test. Entrances into the light were distributed over the 24-h tests, with two peaks of light entrance behavior occurring at about 10 p.m. and 6 a.m.

When striate cortex was removed either with large lesions, including all of the posterior neocortex, or smaller restricted lesions, there were no significant changes in avoidance behavior from preoperative scores $(t=0.85, p>.05$; Table 1$)$. The behavior of individual animals across conditions was also highly similar, yielding a preoperative-postoperative correlation of 0.83 . There was no significant difference in behavior between the animals with large and small neocortical lesions, and so they were grouped together for analysis. Examples of

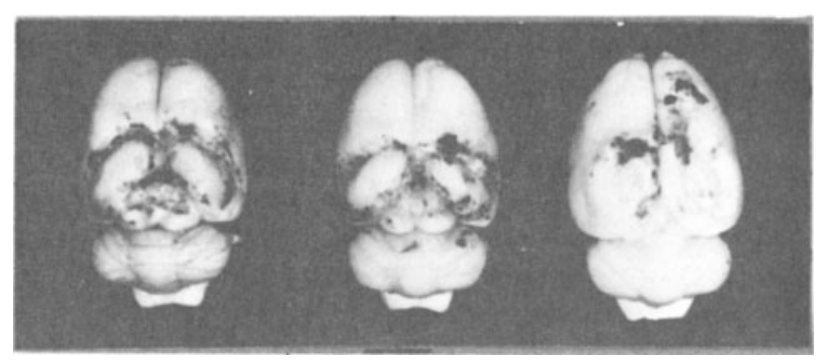

Fig. 2. Dorsal view of three representative lesions produced by the suction technique. (A) Posterior neocortex lesion plus superior colliculus lesion. (B) Posterior neocortex lesion. (C) Lesion restricted to posterior visual cortex (Area 17). For an excellent description of degeneration in the lateral geniculate body following identical lesions, see Bland and Cooper (1970). 

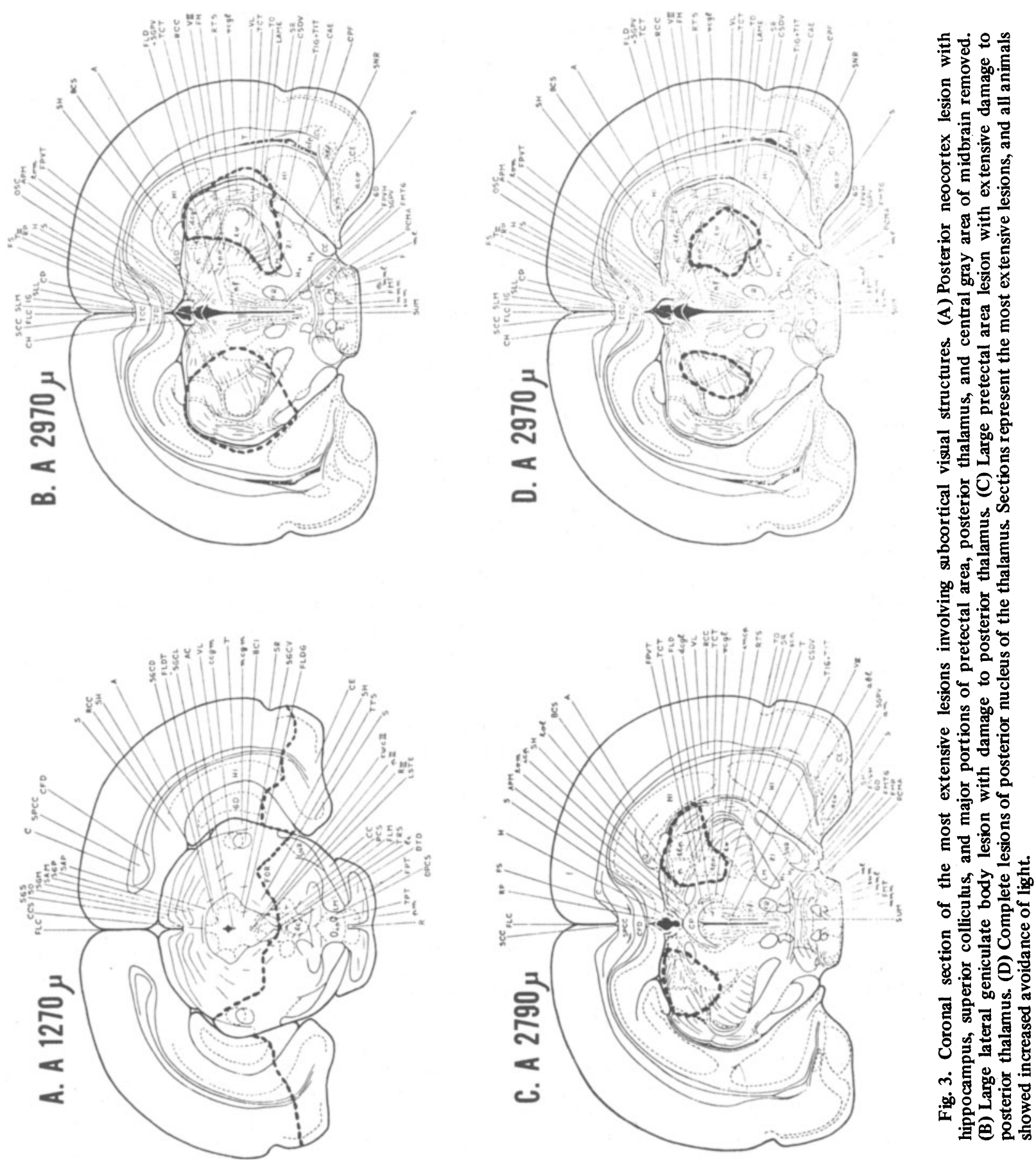

the extent of total posterior cortex lesions are shown in Fig. 2.

Results from destruction in other visual centers using the electrolytic method indicated that superior colliculus lesions $(\mathrm{N}=8,165 \pm 189$ vs $122 \pm 99, \mathrm{t}=1.08, \mathrm{p}>.05)$ and pretectial lesions $(\mathrm{N}=11,112 \pm 94$ vs $84 \pm 914, \mathrm{t}=$ $0.75, p>.05)$ produced no significant effect on light-avoidance behavior, but lesions in the posterior nucleus of the thalamus (NTP) $(\mathrm{N}=8,168$ vs $38 \pm 38$, $\mathrm{t}$ $=4.03, \mathrm{p}<.05)$ and lateral geniculate body (LGB)
$(\mathrm{N}=7, \quad 150 \pm 130$ vs $33 \pm 35, \mathrm{t}=3.52, \mathrm{p}<.05)$ increased light avoidance significantly. Animals with NTP lesions often failed to enter the lighted compartment at all on many days. These animals all showed near total dilation of the pupils and loss of the constriction response to bright light. Histological analysis (Fig. 3) indicated that animals with the most complete LGB destruction also suffered some damage to the NTP area and some, but not total, loss of the constriction reflex, thus it is possible that the increased 
Table 1

Mean Daily Time in Dark, Amount Eaten in Dark, Defecated in Dark, Crosses Between Compartments, and Number of Food Pellets Hoarded from Light to Dark in Two 14-Day Periods for Rats When Normal and Following Striate Cortex Lesions

\begin{tabular}{lcc}
\hline & & Striate \\
Behavior & 89 & 92 \\
Time in Dark (Percent) & 95 & 96 \\
Amount Eaten in Dark (Percent) & 27 & 27 \\
Defecation in Dark (Percent) & 45 & 48 \\
Crosses Between Compartments & 4.5 & 4.4 \\
Food Pellets Hoarded to Dark & \\
\hline
\end{tabular}

avoidance behavior shown by LGB-lesioned animals may have been due to NTP damage rather than LGB damage.

Histological analysis also revealed that even very large electrolytic lesions of the superior colliculus left major portions of that nucleus intact. Therefore, an attempt was made to remove the whole superior colliculus by suction. This technique involved neocortex removal along with SC damage. The combined lesions, rather than reducing the animals' avoidance response to light, significantly increased avoidance of light $(N=10$, $116 \pm 109$ vs $17 \pm 37, t=3.51, p<.01)$. Some of these lesions were found to be extremely large and included damage to the pretectal nuclei as well as portions of the NTP and other nonvisual midbrain structures.

Attempts were made to determine if other telencephalic structures influenced light-avoidance behavior. Frontal cortex lesions $(\mathrm{N}=6)$, olfactory bulb removal $(\mathrm{N}=4)$, and decortication $(\mathrm{N}=10)$ produced no significant changes in avoidance behavior. However, total removal of the hippocampus following posterior neocortex lesions significantly increased light-avoidance behavior $(\mathrm{N}=6,138 \pm 30 \mathrm{~min}$ vs $5 \pm 24 \mathrm{~min}, \mathrm{t}=2.96$, $\mathrm{p}<.05)$. These lesions were accompanied by no obvious abnormal changes in pupillary light response.

\section{Other Behaviors}

Many normal animals were observed to hoard food pellets from the lighter to darker compartment each day as well as to defecate nearly exclusively in the lighted compartment. Thirteen of 16 animals which received striate lesions showed both of these behaviors preoperatively. Following neocortical lesions, these behaviors were still well developed in 12 of the animals. A summary of these results is given in Table 1 . Other lesions nearly always disrupted these behaviors to such an extent that it was not possible to determine whether these behaviors could survive the lesions without significantly increasing the number of animals in the experimental groups. Analysis of the activity of animals showed that rats which showed increases of avoidance behavior showed decreases in the number of daily crosses; however, no significant changes were recorded for other groups from normal preoperative activity levels (about 20-70 crosses per day in different animals, see Table 1).

\section{DISCUSSION}

The main finding of this paper is that primary visual-system lesions do not produce decreases in light-avoidance behavior if animals are tested continuously for a number of days. Increased avoidance behavior followed combined neocortex and superior colliculus lesions, lateral geniculate body lesions, and lesions of the posterior nucleus of the thalamus, while no change followed striate, pretectal, and superior colliculus lesions. Since histological examination of lesion extent indicated that the combined striate and SC lesions and the LGB lesions impinged on the NPT area, it is possible that in these cases increased avoidance behavior results from NPT damage in these groups. However, the results are consistent in indicating that primary visual-center lesions do not reduce light-avoidance behavior as has been suggested by a number of previous studies (Altman, 1962; Bauer, 1968; Horel et al, 1966; Krechevsky, 1936; Parker et al, 1969; Parker \& Treichler, 1973).

Differences in the tasks used by Altman (1962) and Parker and Treichler (1973) and this study consist mainly in the amount of time the animals were left in the test situation, $10-15 \mathrm{~min}$ vs 14 or more days. Animals in the former studies were also more active than control rats, a result not obtained in the present study. It is possible that the lesioned animals showed greater activity in novel situations because they have reduced visual capacities (Bauer \& Cooper, 1967; Goodale \& Cooper, 1965). Altman (1962) suggested increased activity as an alternate explanation for his results. If this interpretation is correct, it would indicate that Altman's (1962) light-avoidance test is not a reliable test of light avoidance, because the time period is too short to allow animals to make discriminations. Changes in light aversion reported in studies using more formal discrimination tasks (Bauer, 1968; Horel et al, 1966; Parker et al, 1969) have been examined elsewhere and attributed to specific variables in the tasks used (Parker \& Treichler, 1973; Stratton, 1971; Stratton \& Edwards, 1971).

Tests of such long duration were not necessary to show the present effects, since preliminary tests lasting 1-2 days indicated that identical results would have been obtained. The longer tests produced a number of findings that have not previously been reported. Light-avoidance behavior in normal rats is distributed as a J curve. (Variability in individual animals' avoidance of light has also been reported by Hanson, 1965; and Keller \& Oberlin, 1942.) Most rats spent very little time in the light, but some rats spent as long as $12 \mathrm{~h}$ each day in the light. The behavior of individual rats was also extremely stable across days. Whether the $\mathrm{J}$ distribution reflects emotionality differences in the animals or differences in "aversion" to light is not known. It is unlikely that the results were due to compartment differences, since reversing the compartments or moving the animals from 
one test apparatus to the other did not change the behavior. In addition, blinding by enucleation abolished the differential response, so the effects appear to be determined primarily by illumination differences. Normal rats were found to hoard food from the lighter to the darker compartments, eat nearly exclusively in the darker compartment, but defecate nearly exclusively in the lighter compartment. It was also observed that a number of days passed before hoarding behavior and selective defecatory behavior were displayed. These behaviors were abolished by enucleation, suggesting that they were also determined by illumination differences. None of these behaviors were affected by either large or small posterior neocortex lesions. Hoarding behavior has been reported to survive posterior neocortex lesions in rats (Stamm, 1953), however, selective defecation by rats in an experimental test has not previously been reported.

One of the implications of previous studies (for example, see Altman, 1962) is that light-avoidance behavior reflects activity in central mechanisms controlling light "aversion" and this aversive behavior can be reduced by central visual-system lesions. The present results indicate that such an effect is yet to be demonstrated. NPT lesions did increase avoidance of light, but they also caused a loss of the pupillary constriction response to light. Intense flooding of the retina with light could account for increased avoidance. Whether or not "aversion" was changed could be tested by placing a contact lens with an artificially constricted pupil on the animal's eye. It could be asked if parametric tests with extremes in illumination levels would have given different results. However, avoidance of light by individual rats appears to give a distribution which shows that the test was sensitive to this behavior in rats. In addition, previous studies appear to indicate that lesion effects should occur at all illumination levels. In the case of striate rats, detection thresholds are unchanged by the lesions (Cooper, Freeman, \& Pinel, 1967), which may indicate that parametric analysis would not produce results much different from those reported here.

A question raised by the present results is: What structures in the brain remain sensitive to light to maintain avoidance after the posterior neocortex and superior colliculus were totally aspirated, along with portions of the pretectal area and NPT? It is possible that the LGB, particularly the ventral nuclei, could maintain this behavior. Horel (1968) has suggested that this area may support brightness discrimination following somewhat similar large lesions. Alternately, the finding that LGB lesions do not reduce avoidance behavior may indicate that the accessory optic system (Hayhow, Webb, \& Jarvie, 1960), through hypothalamic or midbrain connections, may control this behavior. It is also possible that avoidance is controlled by structures outside primary visual projection areas. It was found that if the hippocampus was aspirated in conjunction with posterior neocortex, light avoidance increased. Donovick (1968) has also reported light-avoidance enhancement following septal lesions. However, no reports have yet been made of extravisual-system lesions' reducing light avoidance.

\section{REFERENCES}

Abelman, W. H., \& Morgan, C. T. Effects of cortical lesions upon light aversion in the rat. Journal of Comparative Psychology, 1943, 36, 157-168.

Altman, J. Effects of lesions in central nervous visual structures on light aversion in rats. American Journal of Physiology, 1962, 202, $1208-1210$.

Bauer, J. H. Visual and nonvisual behavior of the rat after early andlate posterior neocortical lesions. Doctoral dissertation, Faculty of Graduate Studies and Research, University of Manitoba, 1968.

Bauer, J. H., \& Cooper, R. M. Effects of posterior cortical lesions on performance of a brightness-discrimination task. Journal of Comparative \& Phy siological Psychology, 1967, 64, 84-93.

Bland, B. H., \& Cooper, R. M. Experience and vision of the posterior neodecorticate rat. Physiology \& Behavior, 1970, 5, 211-214.

Cooper, R. M., Freeman, I., \& Pinel, J. P. J. Absolute thresh old of vision in the rat after removal of striate cortex. Journal of Comparative \& Physiological Psychology, 1967, 64, 36-39.

Crozier, W. J., \& Pincus, G. Phototropism in young rats. Journal of Genetic Psychology, 1926-1927, 10, 407-417.

Donovick, P. J. Effects of localized septal lesions on hippocampal EEG activity and behavior in rats. Journal of Comparative \& Physiological Psychology, 1968, 66, 569-578.

Goodale, M., \& Cooper, R. M. Cues utilized by normal and posterior neodecorticate rats in the Yerkes brightness discrimination task. Psychonomic Science, 1965, 3, 513-514.

Hanson, L. F. Light avoidance and light aversion in the albino rat as a function of intensity of illumination. Doctoral dissertation, Faculty of Pure Science, Columbia University, 1965.

Hayhow, W. R., Webb, C., \& Jervie, A. The accessory optic fiber system in the rat. Journal of Comparative Neurology, 1960,

115, 187-215.
Horel, J. A. Effects of subcorticallesions on brightness discrimination acquired by rats without visual cortex. Journal of Comparative \& Physiological Psychology, 1968, 65, 103-109.

Horel, J. A., Bettinger, L. A., Royce, G. J., \& Meyer, R. D. The role of neocortex in the learning and relearning of two visual habits by the rat. Journal of Comparative \& Physiological Psychology, 1966, 61, 66-78.

Keller, F. S. Light-aversion in the white rat. Psychological Record, 1941, 4, 235-250.

Keller, F. S., \& Oberlin, K. W. A simple technique for measuring light-dark preference in the white rat. Journal of Genetic Psychology, 1942, 61, 163-166.

Krechevsky, I. Brain mechanisms and brightness discrimination learning. Journal of Comparative Psychology, 1936, 21, 405-445.

Parker, T. D., Erickson, C. K., \& Treichler, F. R. The influence of brightness preferences on the assessment of cortical lesion deficits in rats. Journal of Comparative \& Physiological Psychology, 1969, 67, 240-244.

Parker, T. D., \& Treichler, R. F. The influence of restricted posterior neodecortication on brightness preference and brightness discrimination performance of rats. Physiology \& Behavior, 1973, 10, 473-478.

Spear, P. D., \& Braun, J. J. Nonequivalence of normal and posterioriy neodecorticated rats on two brightness discrimination problems. Journal of Comparative \& Physiological Psychology, 1969, 2, 235-239.

Stamm, J. S. Effects of cortical lesions on established hoarding activity in rats. Journal of Comparative \& Physiological Psychology, 1953, 46, 299-304.

Stratton, L. O. Effects of dark preference on attention to position cue in posterior neodecorticated albino rats. Psychonomic Science, 1971, 24, 105-106.

Stratton, L. O., \& Edwards, S. Preference and sensory factors in brightness discrimination learning of posterior neodecorticated albino rats. Psychonomic Science, 1971, 24, 9-10.

Teitelbaum, P., \& Epstein, A. N. The lateral hypothalamic syndrome. Psychological Review, 1962, 69, 74-90.

Turner, W. D. The development of perception: I. Visual direction; the first eidoscopic orientations of the albino rat. Journal of Genetic Psychology, 1935, 47, 121-140.

Zeman, W., \& Innes, J. R. Craigie's neuroanatomy of the rat. New York: Academic Press, 1963. accepted February 21, 1974.) 